Pacific

Journal of

Mathematics

THE $W$-ENTROPY FORMULA FOR THE WITTEN LAPLACIAN ON MANIFOLDS WITH TIME DEPENDENT METRICS AND POTENTIALS

SONGZI LI AND XIANG-DoNG LI

Volume 278 No. 1

November 2015 


\title{
THE $W$-ENTROPY FORMULA FOR THE WITTEN LAPLACIAN ON MANIFOLDS WITH TIME DEPENDENT METRICS AND POTENTIALS
}

\author{
SONGZI Li AND XIANG-DONG Li
}

\begin{abstract}
We develop a new approach to prove the $W$-entropy formula for the Witten Laplacian via warped product on Riemannian manifolds, giving a natural geometric interpretation of the quantities appearing in the $W$-entropy formula. We also prove the $W$-entropy formula for the Witten Laplacian on compact Riemannian manifolds with time dependent metrics and potentials, as well as for the backward heat equation associated with the Witten Laplacian on compact Riemannian manifolds equipped with Lott's modified Ricci flow. Our results extend to complete Riemannian manifolds with negative $\boldsymbol{m}$-dimensional Bakry-Émery Ricci curvature, and to compact Riemannian manifolds with $K$-super $\boldsymbol{m}$-dimensional Bakry-Émery Ricci flow. As an application, we prove that the optimal logarithmic Sobolev constant on compact manifolds equipped with the $K$-super $m$-dimensional BakryÉmery Ricci flow is decreasing in time.
\end{abstract}

\section{Introduction}

Let $M$ be a complete Riemannian manifold with a fixed Riemannian metric $g$ and a fixed potential $\phi \in C^{2}(M)$. Let $d \mu=e^{-\phi} d v$, where $d v$ is the Riemannian volume measure on $(M, g)$. The Witten Laplacian (also called the weighted Laplacian),

$$
L=\Delta-\nabla \phi \cdot \nabla
$$

is a self-adjoint and nonnegative operator on $L^{2}(M, \mu)$. By Itô's calculus, one can construct the symmetric diffusion process $X_{t}$ associated to the Witten Laplacian by solving the SDE

$$
d X_{t}=\sqrt{2} d W_{t}-\nabla \phi\left(X_{t}\right) d t
$$

S. Li was partially supported by the China Scholarship Council. X.-D. Li was supported by NSFC No. 11371351, Key Laboratory of Random Complex Structures and Data Science, AMSS, CAS, No. 2008DP173182, and a Hundred Talents Project of AMSS, CAS. MSC2010: primary 53C44, 58J35, 58J65; secondary 60J60, 60H30.

Keywords: Bakry-Émery Ricci curvature, $W$-entropy, Witten Laplacian, modified Ricci flow. 
where $W_{t}$ is the Brownian motion on $M$. Moreover, it is well known that the transition probability density function of the diffusion process $X_{t}$ is exactly the fundamental solution to the heat equation of $L$, i.e., the heat kernel of the Witten Laplacian $L$. In view of this, it is a fundamental problem to study the heat equation and related properties for the Witten Laplacian on manifolds.

In recent years, important progress has been made in the study of the heat equation associated with the Witten Laplacian by using new ideas and new methods from geometric analysis, PDEs and probability theory. In particular, F. Otto [2001] introduced an infinite dimensional Riemannian structure on the Wasserstein space of probability measures on $\mathbb{R}^{n}$ and proved that the heat equation

$$
\partial_{t} u=L u
$$

can be realized as the reverse gradient flow of the Boltzmann-Shannon entropy ${ }^{1}$

$$
H(u)=-\int_{M} u \log u d \mu .
$$

See also [Otto and Villani 2000; Sturm 2005; von Renesse and Sturm 2005; Villani 2003; 2009] for the extension of Otto's work to Riemannian manifolds.

The Witten Laplacian is a natural extension of the standard Laplace-Beltrami operator and has a close connection to differential geometry, probability theory, quantum field theory and statistical mechanics. In view of this, it is natural to raise the question whether one can extend the results which hold for the standard LaplaceBeltrami operator to the Witten Laplacian on manifolds. The main tool which makes such an extension possible is the so-called Bakry-Émery Ricci curvature associated to $L$ [Bakry and Émery 1985],

$$
\operatorname{Ric}(L)=\operatorname{Ric}+\nabla^{2} \phi,
$$

which plays the same role as the Ricci curvature for the standard Laplace-Beltrami operator. We refer the reader to [Bakry and Qian 1999; Bakry and Ledoux 2006; Li 2005] for the Li-Yau Harnack estimates and the heat kernel estimates to the heat equation (1), and to [Li 2005] for the extension of S.-T. Yau's Strong Liouville theorem for the positive $L$-harmonic functions and the $L^{1}$-uniqueness of the heat equation on complete Riemannian manifolds. See also [Andrews and Ni 2012; Bakry and Qian 2000; Fang et al. 2009; Futaki et al. 2013; Otto and Villani 2000; von Renesse and Sturm 2005; Villani 2003; 2009; Wei and Wylie 2009] for other results on the Witten Laplacian and Bakry-Émery Ricci curvature on manifolds with weighted measures.

\footnotetext{
${ }^{1}$ Equivalently, the heat equation (1) is the gradient flow of $\operatorname{Ent}(u)=-H(u)$ on the Wasserstein space $\mathscr{P}_{2}\left(\mathbb{R}^{n}\right)$ equipped with Otto's infinite dimensional Riemannian metric.
} 
The Bakry-Émery Ricci curvature has been essentially used in Perelman's work on the entropy formula for Ricci flow. Perelman [2002] first introduced the FFfunctional on the space of Riemannian metrics and smooth functions, i.e., $M=$ $\{$ Riemannian metric $g$ on $M\} \times C^{\infty}(M)$, as follows:

$$
\mathscr{F}(g, f)=\int_{M}\left(R+|\nabla f|^{2}\right) e^{-f} d v,
$$

where $f \in C^{\infty}(M), R$ denotes the scalar curvature on $(M, g)$, and $d v$ denotes the volume measure. Under the constraint condition which requires that

$$
d m=e^{-f} d v
$$

is a fixed weighted measure on $(M, g)$, Perelman proved that the gradient flow of $\mathscr{F}_{F}$ with respect to the standard $L^{2}$-metric on $M$ is given by the modified Ricci flow

$$
\partial_{t} g=-2\left(\operatorname{Ric}+\nabla^{2} f\right),
$$

and $f$ satisfies the so-called conjugate heat equation

$$
\partial_{t} f=-\Delta f-R .
$$

Moreover, Perelman [2002] introduced the $W$-entropy and proved its monotonicity for the Ricci flow on compact manifolds. This result plays an important role in the proof of the no local collapsing theorem and in the final resolution of the Poincare and geometrization conjectures (see also [Cao and Zhu 2006; Morgan and Tian 2007; Kleiner and Lott 2008]). Since then, many people have derived the $W$-entropy formula for various geometric evolution equations and used it to study further analysis and geometric properties of manifolds. See, e.g., [Chow et al. 2006; Chang et al. 2011; Kleiner and Lott 2008; Ni 2004a; 2004b; Ecker 2007; Lu et al. 2009; Kotschwar and Ni 2009].

In [Li 2012] (see also [Li 2007; 2011; 2014]), inspired by Perelman's work on the $W$-entropy formula for Ricci flow, the second author proved the $W$-entropy formula for the fundamental solution of the Witten Laplacian on complete Riemannian manifolds with the bounded geometry condition. This extends a previous result due to Ni [2004b; 2004a], who proved an analogue of Perelman's $W$-entropy formula for the heat equation $\partial_{t} u=\Delta u$ on complete Riemannian manifolds with a fixed metric. More precisely, we have:

Theorem 1.1 [Li 2007; 2012; 2011; 2014]. Let (M,g) be a compact Riemannian manifold, or a complete Riemannian manifold with the bounded geometry condition, ${ }^{2}$ and $\phi \in C^{4}(M)$ with $\nabla \phi \in C_{b}^{3}(M)$. Let $m \geq n$, and $u=e^{-f} /(4 \pi t)^{m / 2}$ be a positive solution of the heat equation $\partial_{t} u=L u$ when $M$ is compact, or the

\footnotetext{
${ }^{2}$ We say that $(M, g)$ satisfies the bounded geometry condition if the Riemannian curvature tensor Riem and its covariant derivatives $\nabla^{k}$ Riem are uniformly bounded on $M, k=1,2,3$.
} 
fundamental solution associated with the Witten Laplacian, i.e., the heat kernel to the heat equation $\partial_{t} u=L u$, when $M$ is complete. Let

$$
H_{m}(u, t)=-\int_{M} u \log u d \mu-\frac{m}{2}(1+\log (4 \pi t)) .
$$

Define

$$
W_{m}(u, t)=\frac{d}{d t}\left(t H_{m}(u)\right)
$$

Then

$$
W_{m}(u, t)=\int_{M}\left(t|\nabla f|^{2}+f-m\right) \frac{e^{-f}}{(4 \pi t)^{m / 2}} d \mu
$$

and

$$
\begin{aligned}
\frac{d}{d t} W_{m}(u, t)=-2 \int_{M} t\left(\left|\nabla^{2} f-\frac{g}{2 t}\right|^{2}\right. & \left.+\operatorname{Ric}_{m, n}(L)(\nabla f, \nabla f)\right) u d \mu \\
& -\frac{2}{m-n} \int_{M} t\left(\nabla \phi \cdot \nabla f+\frac{m-n}{2 t}\right)^{2} u d \mu,
\end{aligned}
$$

where

$$
\operatorname{Ric}_{m, n}(L)=\operatorname{Ric}+\nabla^{2} \phi-\frac{\nabla \phi \otimes \nabla \phi}{m-n}
$$

is the m-dimensional Bakry-Émery Ricci curvature associated with the Witten Laplacian $L$.

In particular, if $(M, g, \phi)$ is compact or satisfies the bounded geometry condition and $\operatorname{Ric}_{m, n}(L) \geq 0$, then the $W$-entropy is decreasing in time $t$, i.e.,

$$
\frac{d}{d t} W_{m}(u, t) \leq 0, \quad \text { for all } t \geq 0 .
$$

The purpose of this paper is to extend the $W$-entropy formula in Theorem 1.1 to the heat equation (1) associated with the time dependent Witten Laplacian on compact Riemannian manifolds equipped with time dependent metrics and potentials. In view of Perelman's work using the $W$-entropy formula for the Ricci flow to remove "the major stumbling block in Hamilton's approach to geometrization" [Perelman 2002], it might be possible that the $W$-entropy formula for the time dependent Witten Laplacian can bring some new insights to the study of geometric analysis on Riemannian manifolds with time dependent metrics and potentials. Our results can be regarded as the $m$-dimensional analogue of Perelman's results for the Ricci flow, where the Ricci curvature for the Ricci flow is replaced by the $m$-dimensional BakryÉmery Ricci curvature, and the Laplacian is replaced by the Witten Laplacian.

We are now in a position to state the main results of this paper. 
Theorem 1.2. Let $(M, g(t), t \in[0, T])$ be a family of compact Riemannian manifolds with potential functions $\phi(t) \in C^{\infty}(M), t \in[0, T]$. Suppose that $g(t)$ and $\phi(t)$ satisfy the conjugate equation

$$
\frac{\partial \phi}{\partial t}=\frac{1}{2} \operatorname{Tr}\left(\frac{\partial g}{\partial t}\right)
$$

Let

$$
L=\Delta_{g(t)}-\nabla_{g(t)} \phi(t) \cdot \nabla_{g(t)}
$$

be the time dependent Witten Laplacian on $(M, g(t), \phi(t))$. Let $u$ be a positive solution of the heat equation

$$
\partial_{t} u=L u
$$

with initial data $u(0)$ satisfying $\int_{M} u(0) d \mu(0)=1$. Let

$$
H_{m}(u, t)=-\int_{M} u \log u d \mu-\frac{m}{2}(1+\log (4 \pi t)) .
$$

Define

$$
W_{m}(u, t)=\frac{d}{d t}\left(t H_{m}(u)\right)
$$

Then

$$
W_{m}(u, t)=\int_{M}\left(t|\nabla \log u|^{2}-\log u-\frac{m}{2}(2+\log (4 \pi t))\right) u d \mu,
$$

and

$$
\text { (4) } \begin{aligned}
\frac{d}{d t} W_{m}(u, t)=-2 t & \int_{M}\left|\nabla^{2} \log u+\frac{g}{2 t}\right|^{2} u d \mu \\
-\frac{2 t}{m-n} \int_{M}\left(\nabla \phi \cdot \nabla \log u-\frac{m-n}{2 t}\right)^{2} u d \mu & \quad-2 \int_{M} t\left(\frac{1}{2} \frac{\partial g}{\partial t}+\operatorname{Ric}_{m, n}(L)\right)(\nabla \log u, \nabla \log u) u d \mu .
\end{aligned}
$$

In particular, if $\{g(t), \phi(t), t \in(0, T]\}$ satisfies the $m$-dimensional Perelman super Ricci flow and the conjugate equation

$$
\begin{gathered}
\frac{1}{2} \frac{\partial g}{\partial t}+\operatorname{Ric}_{m, n}(L) \geq 0, \\
\frac{\partial \phi}{\partial t}=\frac{1}{2} \operatorname{Tr}\left(\frac{\partial g}{\partial t}\right),
\end{gathered}
$$

then $W_{m}(u, t)$ is decreasing in $t \in(0, T]$, i.e.,

$$
\frac{d}{d t} W_{m}(u, t) \leq 0, \quad \text { for all } t \in(0, T] .
$$


As an application of the $W$-entropy formula for the Witten Laplacian on manifolds with time dependent metrics and potentials, in the following theorem we prove that the optimal logarithmic Sobolev constant associated with the Witten Laplacian on compact manifolds equipped with the $m$-dimensional Perelman super Ricci flow is decreasing in time.

Theorem 1.3. Let $(M, g(t), \phi(t), t \in[0, T])$ be as in Theorem 1.2. Then, for any fixed $t \in[0, T]$, there exists a positive and smooth function $u=e^{-v / 2}$ such that $v$ achieves the optimal logarithmic Sobolev constant $\mu(t)$ defined by

$$
\mu(t):=\inf \left\{W_{m}(u, t): \int_{M} \frac{e^{-v}}{(4 \pi t)^{m / 2}} d \mu=1\right\} .
$$

Indeed, $u=e^{-v / 2}$ is a solution to the nonlinear PDE

$$
-4 t L u-2 u \log u-m u=\mu(t) u .
$$

Moreover, if $\{g(t), \phi(t), t \in[0, T]\}$ satisfies the $m$-dimensional Perelman super Ricci flow (5) and the conjugate equation (6), then $\mu(t)$ is decreasing in $t$ on $[0, T]$.

Remark 1.4. We believe that, via the approach used in [Li 2012; 2011; 2014], it would be possible to further extend the $W$-entropy formula in Theorem 1.2 to the fundamental solution of the heat equation associated with the Witten Laplacian on complete Riemannian manifolds with time dependent metrics and potentials satisfying the bounded geometry condition. Technically, this would require some Hamilton-type gradient estimates for the logarithm of the heat kernel of the Witten Laplacian on complete Riemannian manifolds with time dependent metrics and potentials satisfying the uniformly bounded geometry condition. ${ }^{3}$ We will study this problem in a forthcoming paper. If this can be verified, we can derive, for a family $\{g(t), \phi(t), t \in(0, T]\}$ of metrics and potentials satisfying (5) and (6) on a complete Riemannian manifold $M$ with the uniformly bounded geometry condition, that

$$
\frac{d}{d t} W_{m}(u, t)=0, \quad \text { for some } t=\tau \in(0, T]
$$

if and only if at time $t=\tau$, we have

$$
\nabla^{2} \log u=-\frac{g}{2 \tau}, \quad \frac{1}{2} \frac{\partial g}{\partial t}+\operatorname{Ric}_{m, n}(L)=0, \quad \text { and } \quad \nabla \phi \cdot \nabla \log u=\frac{m-n}{2 \tau} .
$$

By the same argument as used in $[\mathrm{Li} 2012 ; 2011 ; 2014]$, we can further prove the following rigidity result. Let $\{g(t), \phi(t), t \in(0, T]\}$ be a family of metrics and potentials satisfying (5) and (6) on a complete Riemannian manifold $M$ with the

\footnotetext{
${ }^{3}$ We say that $(M, g(t), \phi(t), t \in[0, T])$ satisfies the uniformly bounded geometry condition if there exists some $N \in \mathbb{N}$ such that for all $\varepsilon \in(0, T)$, the $k$-th order covariant derivatives $\nabla^{k} \operatorname{Riem}(g(t))$ of the Riemannian curvature tensor Riem $(g(t))$, as well as the $k$-th order covariant derivatives $\nabla^{k} \phi(t)$ of $\phi(t)$, are uniformly bounded on $[\varepsilon, T] \times M$ for $k=0, \ldots, N$.
} 
uniformly bounded geometry condition. Let $u$ be the fundamental solution to the heat equation $\partial_{t} u=L u$. Then

$$
\frac{d}{d t} W_{m}(u, t)=0, \quad \text { for some } t=\tau \in(0, T],
$$

if and only if $(M, g(\tau))$ is isometric to $\mathbb{R}^{n}, \phi(\tau)$ is identically equal to a constant, $m=n, \partial g / \partial t=0, \partial \phi / \partial t=0$ at $t=\tau$, and

$$
u(x, \tau)=\frac{e^{-\|x\|^{2} / 4 \tau}}{(4 \pi \tau)^{n / 2}}, \quad \text { for all } x \in M=\mathbb{R}^{n} .
$$

The rest of this paper is organized as follows. In Section 2, we give a new proof of Theorem 1.1. ${ }^{4}$ In Section 3, we prove the dissipation formula of the BoltzmannShannon entropy for the heat equation of the Witten Laplacian on compact manifolds with time dependent metrics and potentials. In Section 4, we prove Theorem 1.2 and Theorem 1.3. In Section 5, we use Perelman's $W$-entropy formula for Ricci flow to derive the $W$-entropy formula for the backward heat equation of the Witten Laplacian on compact Riemannian manifolds equipped with a modified Ricci flow introduced by Lott [2009]. In Section 6, we extend Theorem 1.1 and Theorem 1.2 to the case $\operatorname{Ric}_{m, n}(L) \geq-K$ and compact $K$-super $m$-dimensional Bakry-Émery Ricci flow.

\section{A new proof of Theorem 1.1}

To prove Theorem 1.1, we first recall some elementary geometric formulas on warped product metrics.

Let $m \in \mathbb{N}, m \geq n$. Let $\widetilde{M}=M \times N$, where $\left(N, g_{N}\right)$ is a compact Riemannian manifold with dimension $q=m-n$. Let $\phi \in C^{2}(M)$. We consider the warped product metric

$$
\tilde{g}=g_{M} \oplus e^{-2 \phi / q} g_{N}
$$

on $\tilde{M}$. Let $v_{N}$ be the volume measure on $N$. Then the volume measure on $(\tilde{M}, \tilde{g})$ is given by

$$
d \operatorname{vol}_{\widetilde{M}}=e^{-\phi} d \operatorname{vol}_{M} \otimes d v_{N}
$$

Define

$$
d \mu=e^{-\phi} d \operatorname{vol}_{M} .
$$

Then

$$
d \operatorname{vol}_{\tilde{M}}=d \mu \otimes d v_{N}
$$

${ }^{4}$ One of the advantages of our new proof is that it gives a natural geometric interpretation of the third term appearing in the $W$-entropy formula (3). See Remark 2.2. 
Without loss of generality, we may assume that

$$
v_{N}(N)=1 .
$$

Let $\widetilde{\Gamma}$ be the Christoffel symbol on $(\tilde{M}, \tilde{g})$. By direct calculation, we verify that

$$
\begin{array}{lll}
\widetilde{\Gamma}_{i j}^{k}=\Gamma_{i j}^{k}, & \widetilde{\Gamma}_{\alpha \beta}^{k}=q^{-1} g^{k l} \partial_{l} \phi g_{\alpha \beta}, & \widetilde{\Gamma}_{\alpha \beta}^{\gamma}=\Gamma_{\alpha \beta}^{\gamma}, \\
\widetilde{\Gamma}_{i j}^{\alpha}=0, & \widetilde{\Gamma}_{i \alpha}^{k}=0, & \widetilde{\Gamma}_{i \alpha}^{\beta}=0 .
\end{array}
$$

Let $\widetilde{\nabla}$ be the Levi-Civita connection on $(\tilde{M}, \tilde{g})$. For any $f \in C^{2}(M)$, using the formulas

$\widetilde{\nabla}_{i j}^{2} f=\partial_{i} \partial_{j} f-\widetilde{\Gamma}_{i j}^{k} \partial_{k} f, \quad \widetilde{\nabla}_{i \alpha}^{2} f=\partial_{i} \partial_{\alpha} f-\widetilde{\Gamma}_{i \alpha}^{k} \partial_{k} f, \quad \widetilde{\nabla}_{\alpha \beta}^{2} f=\partial_{\alpha} \partial_{\beta} f-\widetilde{\Gamma}_{\alpha \beta}^{k} \partial_{k} f$, we have

$$
\begin{aligned}
\widetilde{\nabla}_{i j}^{2} f & =\nabla_{i j}^{2} f, \\
\widetilde{\nabla}_{\alpha \beta}^{2} f & =-q^{-1} g^{k l} \partial_{l} \phi \partial_{k} f g_{\alpha \beta}, \\
\nabla_{i \alpha}^{2} f & =0 .
\end{aligned}
$$

Hence

$$
\begin{aligned}
\left|\widetilde{\nabla}^{2} f-\frac{\tilde{g}}{2 t}\right|^{2} & =\left|\nabla^{2} f-\frac{g}{2 t}\right|^{2}+\left|\widetilde{\nabla}_{\alpha \beta}^{2} f-\frac{g_{\alpha \beta}}{2 t}\right|^{2} \\
& =\left|\nabla^{2} f-\frac{g}{2 t}\right|^{2}+\left|\frac{g^{k l} \partial_{l} \phi \partial_{k} f g_{\alpha \beta}}{q}+\frac{g_{\alpha \beta}}{2 t}\right|^{2} \\
& =\left|\nabla^{2} f-\frac{g}{2 t}\right|^{2}+\left|\left(\frac{\nabla \phi \cdot \nabla f}{m-n}+\frac{1}{2 t}\right) g_{\alpha \beta}\right|^{2} \\
& =\left|\nabla^{2} f-\frac{g}{2 t}\right|^{2}+\frac{1}{m-n}\left(\nabla \phi \cdot \nabla f+\frac{m-n}{2 t}\right)^{2} .
\end{aligned}
$$

The following result was obtained in a private discussion between Bing-Long Chen and the second author in January 2006.

Theorem 2.1. The Laplace-Beltrami operator on $(\tilde{M}, \tilde{g})$ is given by

$$
\Delta_{\tilde{M}}=L+e^{-2 \phi /(m-n)} \Delta_{N} .
$$

Proof. The proof can be given by a direct calculation.

Proof of Theorem 1.1. To avoid technical issues, we only consider the case of compact manifolds. Let $u=e^{-f} /(4 \pi t)^{m / 2}: M \rightarrow[0, \infty)$ be a positive solution to the heat equation $\partial_{t} u=L u$. Then it satisfies the heat equation

$$
\partial_{t} u=\Delta_{\tilde{M}} u
$$


on $(\tilde{M}, \tilde{g})$. Since $f$ depends only on the variable in the $M$-direction, we have $\widetilde{\nabla} f=\nabla f$. Therefore, the $W$-entropy functional $W_{m}(u, t)$ defined by (2) coincides with the $W$-entropy functional

$$
\widetilde{W}_{m}(u, t)=\int_{\widetilde{M}}\left[t|\widetilde{\nabla} f|^{2}+f-m\right] \frac{e^{-f}}{(4 \pi t)^{m / 2}} d \operatorname{vol}_{\widetilde{M}}
$$

defined on $(\tilde{M}, \tilde{g})$. Applying to $(\tilde{M}, \tilde{g})$ the $W$-entropy formula for the heat equation $\partial_{t} u=\Delta u$ on compact Riemannian manifolds with fixed metrics due to Ni [2004b; 2004a], we have

$$
\frac{d}{d t} \widetilde{W}_{m}(u, t)=-2 \int_{\widetilde{M}} t\left(\left|\widetilde{\nabla}^{2} f-\frac{\tilde{g}}{2 t}\right|^{2}+\widetilde{\operatorname{Ric}}(\widetilde{\nabla} \log u, \widetilde{\nabla} \log u)\right) u d \mu d v_{N}
$$

By (11), we have

$$
\left|\widetilde{\nabla}^{2} f-\frac{\tilde{g}}{2 t}\right|^{2}=\left|\nabla^{2} f-\frac{g}{2 t}\right|^{2}+\frac{2}{m-n}\left(\nabla \phi \cdot \nabla f+\frac{m-n}{2 t}\right)^{2} .
$$

On the other hand, by [Besse 1987; Lott 2003; Li 2005], we have

$$
\widetilde{\operatorname{Ric}}(\widetilde{\nabla} \log u, \widetilde{\nabla} \log u)=\operatorname{Ric}_{m, n}(L)(\nabla \log u, \nabla \log u) .
$$

From (13), (14) and (15), we obtain (3). This finishes the new proof of Theorem 1.1 in the case of compact manifolds.

Remark 2.2. One of the advantages of the above proof is that when $m \in \mathbb{N}$ and $m>n$, the quantity

$$
\frac{1}{m-n}\left(\nabla \phi \cdot \nabla f+\frac{m-n}{2 t}\right)^{2}
$$

appearing in the $W$-entropy formula in Theorem 1.1 has a natural geometric interpretation. It corresponds to the vertical component of the quantity $\left|\widetilde{\nabla}^{2} f-\tilde{g} / 2 t\right|^{2}$ on the warped product manifold $\widetilde{M}=M \times N$ equipped with the metric

$$
\tilde{g}=g \oplus e^{-2 \phi /(m-n)} g_{N} .
$$

In the case where $(M, g)$ is a complete Riemannian manifold with the bounded geometry condition, similarly to [Lott 2003; Charalambous and Lu 2015], by introducing a sequence of warped product metrics $\left\{\tilde{g}_{\varepsilon}\right\}$ on $\widetilde{M}=M \times N$ defined by

$$
\tilde{g}_{\varepsilon}=g \oplus \varepsilon^{2} e^{-2 \phi /(m-n)} g_{N},
$$

and using the fact that the heat kernel of the Laplace-Beltrami $\Delta_{\left(\widetilde{M}, \tilde{g}_{\varepsilon}\right)}$ on $\left(\tilde{M}, \tilde{g}_{\varepsilon}\right)$ (with renormalized volume measure) converges in the $C^{2, \alpha} \cap W^{2, p}$-topology to the heat kernel of the Witten Laplacian $L=\Delta_{M}-\nabla \phi \cdot \nabla$ on $(M, g, \mu)$, we can use the same approach as in the compact case to give a new proof of the $W$-entropy formula for the heat kernel of the Witten Laplacian on complete Riemannian manifolds 
satisfying the bounded geometry condition in Theorem 1.1. We will study this problem in detail in the future.

Remark 2.3. We would like to mention that, after the first version of this paper [ $\mathrm{Li}$ and Li 2014b] was posted online in March 2013, N. Charalambous and Z. Lu posted a preprint [2015] in which they used the warped product approach to prove the Li-Yau differential Harnack inequality on complete Riemannian manifolds with weighted volume measure. Recently we also found a paper by H. Guo, R. Philipowski and A. Thalmaier [2015], in which they studied the Boltzmann entropy dissipation formula on manifolds with time dependent metrics. We would also like to point out that G. Huang and H. Li [2014] extended the $W$-entropy formula for the heat equation of the Witten Laplacian in Theorem 1.1 to the porous medium equation for the Witten Laplacian on compact Riemannian manifolds with fixed metric and potential. We can use the same method developed in Section 2 to give a new proof of their result. See also related works of Y.-Z. Wang et al. [2013; 2014].

\section{Dissipation formula of the Boltzmann-Shannon entropy}

Let $(M, g(t), \phi(t))$ be as in Theorem 1.2. Following [Bakry and Émery 1985; Lott 2003; Li 2005], we introduce the Bakry-Émery Ricci curvature associated with $L$ as

$$
\operatorname{Ric}(L)=\operatorname{Ric}+\nabla^{2} \phi .
$$

The purpose of this section is to prove the following dissipation formula for the Boltzmann-Shannon entropy associated with the Witten Laplacian on manifolds with time dependent metrics and potentials.

Theorem 3.1. Let $u$ be a positive solution to the heat equation $\partial_{t} u=L u$. Let

$$
H(u, t)=-\int_{M} u \log u d \mu
$$

be the Boltzmann-Shannon entropy associated with the Witten Laplacian L. Then

$$
\begin{aligned}
& \frac{\partial^{2}}{\partial t^{2}} H(u, t) \\
& \quad=-2 \int_{M}\left(\left|\nabla^{2} \log u\right|^{2}+\left(\frac{1}{2} \frac{\partial g}{\partial t}+\operatorname{Ric}(L)\right)(\nabla \log u, \nabla \log u)\right) u d \mu .
\end{aligned}
$$

Proof. By direct calculation, we have

$$
\frac{\partial}{\partial t} H(u, t)=-\int_{M} \partial_{t} u(\log u+1) d \mu=-\int_{M} L u(\log u+1) d \mu .
$$

Integrating by parts yields

$$
\frac{\partial}{\partial t} H(u, t)=\int_{M}|\nabla \log u|_{g(t)}^{2} u d \mu
$$


which further implies that, as $\partial_{t}(d \mu)=0$, we have

$$
\begin{aligned}
\frac{\partial^{2}}{\partial t^{2}} H(u, t) & =\int_{M} \frac{\partial}{\partial t}\left(|\nabla \log u|_{g(t)}^{2} u\right) d \mu \\
& =\int_{M}\left[\frac{\partial g i j}{\partial t} \nabla_{i} \log u \nabla_{j} \log u\right] u d \mu+\int_{M} \frac{\partial}{\partial t}\left[\frac{|\nabla u|^{2}}{u}\right]_{g(t) \text { fixed }} d \mu \\
& =\int_{M}\left[-\frac{\partial g_{i j}}{\partial t} \nabla_{i} \log u \nabla_{j} \log u\right] u d \mu+\int_{M} \frac{\partial}{\partial t}\left[\frac{|\nabla u|^{2}}{u}\right]_{g(t) \text { fixed }} d \mu \\
& =\int_{M}\left(-\frac{\partial g}{\partial t}(\nabla \log u, \nabla u)+\frac{\partial}{\partial t}\left[\frac{|\nabla u|^{2}}{u}\right]_{g(t) \text { fixed }}\right) d \mu
\end{aligned}
$$

where $[\cdot]_{g(t)}$ fixed means that the quantity $|\nabla u|^{2}$ in $[\cdot]$ is defined under a fixed metric $g(t)$. We have also used the facts $|\nabla \log u|^{2}=g^{i j} \nabla_{i} \log u \nabla_{j} \log u$ and $\partial_{t} g^{i j}=-\partial_{t} g_{i j}$.

By the entropy dissipation formula in [Bakry and Émery 1985; Li 2014], we have

$$
\begin{aligned}
\int_{M} \frac{\partial}{\partial t}\left[\frac{|\nabla u|^{2}}{u}\right]_{g(t) \text { fixed }} & d \mu \\
& =-2 \int_{M}\left[\left|\nabla^{2} \log u\right|^{2}+\operatorname{Ric}(L)(\nabla \log u, \nabla \log u)\right] u d \mu .
\end{aligned}
$$

Combining (17) and (18) completes the proof of Theorem 3.1.

As an easy consequence of Theorem 1.2, we have the following corollary.

Corollary 3.2. Let $(M, g(t))$ be a closed manifold with a potential $\phi(t)$. Suppose that $(g(t), \phi(t))$ satisfies the Perelman super Ricci flow and the conjugate equation:

$$
\frac{\partial g}{\partial t} \geq-2 \operatorname{Ric}(L), \quad \frac{\partial \phi}{\partial t}=\frac{1}{2} \operatorname{Tr}\left(\frac{\partial g}{\partial t}\right) .
$$

Let $u$ be a positive solution to the heat equation $\partial_{t} u=L u$. Then the BoltzmannShannon entropy

$$
H(u, t)=-\int_{M} u \log u d \mu
$$

is concave in time t, i.e.,

$$
\frac{d^{2}}{d t^{2}} H(u, t) \leq 0
$$

\section{Proofs of Theorem 1.2 and Theorem 1.3}

Following [Li 2014], we introduce

$$
W(u, t)=\frac{d}{d t}(t H(u, t)) .
$$

By direct calculation, we can prove the following. 
Proposition 4.1. We have

$$
W(u, t)=\int_{M}\left[t|\nabla \log u|^{2}-\log u\right] u d \mu,
$$

and

(19) $\frac{d}{d t} W(u, t)$

$$
\begin{aligned}
=-2 \int_{M} t\left(\left|\nabla^{2} \log u\right|^{2}+\left(\frac{1}{2} \frac{\partial g}{\partial t}+\operatorname{Ric}(L)\right)(\nabla\right. & \log u, \nabla \log u)) u d \mu \\
& +2 \int_{M}|\nabla \log u|^{2} u d \mu .
\end{aligned}
$$

Remark 4.2. From (19), we can derive that if

$$
\frac{1}{2} \frac{\partial g}{\partial t}+\operatorname{Ric}(L)-\frac{1}{t} \geq 0,
$$

then

$$
\frac{d}{d t} W(u, t) \leq 0
$$

Let

$$
H_{m}(u, t)=-\int_{M} u \log u d \mu-\frac{m}{2}(1+\log (4 \pi t)) .
$$

Following [Perelman 2002; Ni 2004a; 2004b; Li 2012; 2014], we define $W_{m}(u, t)$ by the Boltzmann entropy formula

$$
W_{m}(u, t)=\frac{d}{d t}\left(t H_{m}(u)\right) .
$$

We can verify that $W_{m}(u, t)$ coincides with the expression given in Theorem 1.2, namely

$$
W_{m}(u, t)=\int_{M}\left(t|\nabla \log u|^{2}-\log u-\frac{m}{2}(2+\log (4 \pi t))\right) u d \mu .
$$

Proof of Theorem 1.2. By (20) and (16) in Theorem 3.1, we have

$$
\begin{array}{r}
\frac{d}{d t} W_{m}(u, t) \\
=-2 \int_{M} t\left(\left|\nabla^{2} \log u\right|^{2}+\left(\frac{1}{2} \frac{\partial g}{\partial t}+\operatorname{Ric}(L)\right)(\nabla \log u, \nabla \log u)\right) u d \mu \\
+2 \int_{M}|\nabla \log u|^{2} u d \mu-\frac{m}{2 t} .
\end{array}
$$

Note that

$$
2 t\left|\nabla^{2} \log u\right|^{2}+\frac{m}{2 t}=2 t\left|\nabla^{2} \log u+\frac{g}{2 t}\right|^{2}+\frac{m-n}{2 t}-2 \Delta \log u .
$$

Hence 


$$
\begin{gathered}
\frac{d}{d t} W_{m}(u, t)=-\frac{m-n}{2 t}-2 t \int_{M}\left|\nabla^{2} \log u+\frac{g}{2 t}\right|^{2} u d \mu \\
+2 \int_{M}|\nabla \log u|^{2} u d \mu+2 \int_{M}(\Delta \log u) u d \mu \\
\quad-2 \int_{M} t\left(\frac{1}{2} \frac{\partial g}{\partial t}+\operatorname{Ric}(L)\right)(\nabla \log u, \nabla \log u) u d \mu .
\end{gathered}
$$

Integrating by parts yields

$$
\begin{aligned}
\int_{M}(\Delta \log u) u d \mu & =\int_{M}(L \log u+\nabla \phi \cdot \nabla \log u) u d \mu \\
& =-\int_{M}|\nabla \log u|^{2} u d \mu+\int_{M}(\nabla \phi \cdot \nabla \log u) u d \mu,
\end{aligned}
$$

whence

$$
\begin{aligned}
\frac{d}{d t} W_{m}(u, t)=-\frac{m-n}{2 t}-2 t \int_{M}\left|\nabla^{2} \log u+\frac{g}{2 t}\right|^{2} u d \mu+2 \int_{M}(\nabla \phi \cdot \nabla \log u) u d \mu \\
-2 \int_{M} t\left(\frac{1}{2} \frac{\partial g}{\partial t}+\operatorname{Ric}(L)\right)(\nabla \log u, \nabla \log u) u d \mu .
\end{aligned}
$$

Note that

$$
\begin{aligned}
& \frac{m-n}{2 t}+2 t \operatorname{Ric}(L)(\nabla \log u, \nabla \log u)-2 \nabla \phi \cdot \nabla \log u \\
& =2 t \operatorname{Ric}_{m, n}(L)(\nabla \log u, \nabla \log u)+\frac{2 t}{m-n}\left(\nabla \phi \cdot \nabla \log u-\frac{m-n}{2 t}\right)^{2} .
\end{aligned}
$$

We conclude that

$$
\begin{array}{r}
\frac{d}{d t} W_{m}(u, t)=-2 t \int_{M}\left|\nabla^{2} \log u+\frac{g}{2 t}\right|^{2} u d \mu-\frac{2 t}{m-n} \int_{M}\left(\nabla \phi \cdot \nabla \log u-\frac{m-n}{2 t}\right)^{2} u d \mu \\
-2 \int_{M} t\left(\frac{1}{2} \frac{\partial g}{\partial t}+\operatorname{Ric}_{m, n}(L)\right)(\nabla \log u, \nabla \log u) u d \mu .
\end{array}
$$

This proves the $W$-entropy formula in Theorem 1.2, and the monotonicity result follows.

Proof of Theorem 1.3. The proof is similar to the one used by Perelman [2002]. See also [Li 2012]. By definition, we have

$$
\mu(t)=\inf _{u}\left\{\int_{M}\left[4 t|\nabla u|^{2}-u^{2} \log u^{2}-m u^{2}\right](4 \pi t)^{-m / 2} d \mu\right\},
$$

where the infimum is taken over all $u$ such that

$$
\int_{M}(4 \pi t)^{-m / 2} u^{2} d \mu=1
$$


Indeed, $\mu(t)$ is the optimal constant in the logarithmic Sobolev inequality stating that for all $u$ satisfying the above condition,

$$
\int_{M} u^{2} \log u^{2}(4 \pi t)^{-m / 2} d \mu \leq \mu(t)+m+4 \int_{M} t|\nabla u|^{2}(4 \pi t)^{-m / 2} d \mu .
$$

By a similar argument as used in [Perelman 2002; Cao and Zhu 2006; Kleiner and Lott 2008; Morgan and Tian 2007], we can prove that the minimization problem (22) has a nonnegative minimizer $u \in H^{1}(M, \mu)$, which satisfies the Euler-Lagrange equation

$$
-4 t L u-2 u \log u-m u=\mu(t) u .
$$

By the regularity theory of elliptic PDEs, we have $u \in C^{1, \alpha}(M)$. By an argument due to Rothaus [1981], we can further prove that $u$ is strictly positive and smooth. Hence $v=-2 \log u$ is also smooth. Moreover, as a consequence of Theorem 1.2, we can derive that $\mu(t)$ is a decreasing function in $t$ on $[0, T]$, provided that $\{g(t), \phi(t), t \in[0, T]\}$ satisfies the $m$-dimensional Perelman super Ricci flow (5) and the conjugate equation (6). This completes the proof of Theorem 1.3.

Remark 4.3. Let $m \in \mathbb{N}$ and $m>n$. Let $\left(N, g_{N}\right)$ be a compact Riemannian manifold of dimension $q=m-n$. Let $\mu=M \times N$ be the product manifold equipped with the time dependent warped product metric

$$
\tilde{g}(t)=g(t) \oplus e^{-2 \phi(t) /(m-n)} g_{N} .
$$

Similarly to Remark 2.2, the quantity

$$
\frac{1}{m-n}\left(\nabla \phi(t) \cdot \nabla \log u-\frac{m-n}{2 t}\right)^{2}
$$

appearing in the $W$-entropy formula in Theorem 1.2 has a natural geometric interpretation. It corresponds to the vertical component of the quantity $\left|\widetilde{\nabla}^{2} \log u+\tilde{g}(t) / 2 t\right|^{2}$ on $(\mathcal{M}, \tilde{g}(t))$.

Remark 4.4. Perelman [2002] gave an interpretation of the $W$-entropy using the Boltzmann entropy formula from statistical mechanics. In [Li 2012; 2011], the second author gave a probabilistic interpretation of the $W$-entropy for the Ricci flow, the heat equation of the Witten Laplacian and the Fokker-Planck heat equation. Note that, as in [Li 2012; 2011; 2014], we have

$$
H_{m}(u, t)=H(u, t)-H(\gamma, t)
$$

where $H(u, t)$ is the Boltzmann-Shannon entropy associated with the heat equation to the Witten Laplacian on $(M, g(t), \phi(t))$, and $H(\gamma, t)$ is the Boltzmann-Shannon entropy of the Gaussian heat kernel $\gamma(x, t)$ on $\mathbb{R}^{m}$ for $m \in \mathbb{N}$ with $m \geq n$,

$$
\gamma(x, t)=\frac{1}{(4 \pi t)^{m / 2}} e^{-\|x\|^{2} / 4 t}, \quad x \in \mathbb{R}^{m}, t>0 .
$$


Thus, in view of its definition (20), the $W$-entropy $W_{m}(u, t)$ can be regarded as the byproduct of the Boltzmann-Shannon entropy. This gives a probabilistic interpretation of the $W$-entropy $W_{m}(u, t)$.

On the other hand, similarly to [Perelman 2002], we can also give a heuristic interpretation of the $W$-entropy using the Boltzmann entropy formula from statistical mechanics. Suppose that there exists a canonical ensemble with a "density of state measure" $g(E) d E$ such that the partition function $Z_{\beta}=\int_{\mathbb{R}^{+}} e^{-\beta E} g(E) d E$ is given by

$$
\log Z_{\beta}=H_{m}(u, t),
$$

where $t=\beta^{-1}$. (Here, as in [Perelman 2002], we do not discuss the issue of whether such a "density of state measure" exists or not.) Then, formally applying the Boltzmann entropy formula from statistical mechanics, the thermodynamical entropy of this canonical ensemble is given by

$$
S=\log Z_{\beta}-\beta \frac{\partial}{\partial \beta} \log Z_{\beta} .
$$

Using the fact $\frac{\partial}{\partial \beta}=\frac{\partial}{\partial t} \frac{\partial t}{\partial \beta}=-\frac{1}{\beta^{2}} \frac{\partial}{\partial t}=-t^{2} \frac{\partial}{\partial t}$, we can prove

$$
S=W_{m}(u, t) .
$$

Moreover, formally using the formula

$$
\frac{d S}{d \beta}=-\beta \frac{\partial^{2}}{\partial \beta^{2}} \log Z_{\beta},
$$

from statistical mechanics, we can reprove the $W$-entropy formula in Theorem 1.2.

\section{The $W$-entropy for the Ricci flow on warped product manifolds}

Let $m \in \mathbb{N}$ and $m \geq n$. Let $\mathbb{T}^{q}$ be the $q$-dimensional torus with a fixed flat metric given in local coordinates by $\sum_{i=1}^{q} d x_{i}^{2}$, where $q=m-n$. Let $\tilde{M}=M \times \mathbb{T}^{q}$ be equipped with a time dependent warped product metric

$$
\tilde{g}(t)=\sum_{i, j=1}^{n} g_{i j}(t) d x^{i} d x^{j}+u(t)^{2 / q} \sum_{\alpha=1}^{q} d x_{\alpha}^{2} .
$$

Lott [2009] studied the Ricci flow $\tilde{g}(t)$ on the warped product manifold $\widetilde{M}=$ $M \times \mathbb{\rrbracket}^{q}$, which consists of a modified Ricci flow for the Riemannian metric $g(t)$ and a forward heat equation for a potential function $\psi(t)=-\log u(t)$ on the manifold $M$. In this section, we use Perelman's $W$-entropy formula for the Ricci flow $\tilde{g}(t)$ on the warped product manifold $\tilde{M}$ to derive the $W$-entropy formula for the backward heat equation associated with the Witten Laplacian $L=\Delta_{g(t)}-\nabla_{g(t)} \psi(t) \cdot \nabla_{g(t)}$ 
on the compact manifold $M$ equipped with Lott's modified Ricci flow $g(t)$ and the time dependent potential $\psi(t)$.

We first recall Lott's Ricci flow on $\widetilde{M}=M \times \mathbb{T}^{q}$. Let $u=e^{-\psi}$. Let $\widetilde{\text { Ric }}$ be the Ricci curvature on $(\tilde{M}, \tilde{g})$, and Ric the Ricci curvature on $(M, g)$. By calculation on warped product manifolds [Besse 1987; Lott 2003; 2009], we have

$$
\widetilde{\operatorname{Ric}}=\operatorname{Ric}_{\psi}^{q}+\frac{1}{q}\left(\Delta \psi-|\nabla \psi|^{2}\right) u^{2 / q} \sum_{i=1}^{q} d x_{i}^{2},
$$

where $\operatorname{Ric}_{\psi}^{q}$ is the $m$-dimensional Bakry-Émery Ricci curvature on $(M, g)$ with respect to the potential function $\psi$, i.e.,

$$
\operatorname{Ric}_{\psi}^{q}=\operatorname{Ric}+\text { Hess } \psi-\frac{1}{q} \nabla \psi \otimes \nabla \psi .
$$

See [Bakry and Émery 1985; Li 2012; 2011; 2014]. Below we will also use the notation $\operatorname{Ric}_{q}$ to denote $\widetilde{R i c}$. By (24), the scalar curvature $R_{q}$ on $(\tilde{M}, \tilde{g})$ is given by

$$
R_{q}=R+2 \Delta \psi-\left(1+\frac{1}{q}\right)|\nabla \psi|^{2}
$$

The Ricci flow on $\tilde{M}$ is defined by

$$
\partial_{t} \tilde{g}=-2 \widetilde{\text { Ric }}
$$

According to [Lott 2009], the Ricci flow equation (25) is equivalent to the equations

$$
\begin{aligned}
\partial_{t} g & =-2 \operatorname{Ric}_{\psi}^{q}, \\
\partial_{t} \psi & =\Delta \psi-|\nabla \psi|^{2} .
\end{aligned}
$$

Note that the first equation (26) is indeed a modified Ricci flow equation for the metric $g(t)$ on $M$, and the second one (27) is a forward heat equation for the potential function $\psi(t)$ on $(M, g(t))$. The systems (26) and (27) are different from Perelman's (modified) Ricci flow and the conjugate heat equation introduced in [Perelman 2002], i.e.,

$$
\begin{aligned}
& \frac{\partial g}{\partial t}=-2\left(\operatorname{Ric}+\nabla^{2} f\right), \\
& \frac{\partial f}{\partial t}=-\Delta f-R,
\end{aligned}
$$

and are also different from the $m$-dimensional Perelman Ricci flow and the conjugate heat equation

$$
\begin{aligned}
& \frac{\partial g}{\partial t}=-2\left(\text { Ric }+\nabla^{2} f-\frac{\nabla f \otimes \nabla f}{m-n}\right), \\
& \frac{\partial f}{\partial t}=-\Delta f+\frac{|\nabla f|^{2}}{m-n}-R .
\end{aligned}
$$


Let $\phi$ be a positive solution to the conjugate heat equation on $(\tilde{M}, \tilde{g})$,

$$
\partial_{t} \phi=-\Delta_{\tilde{M}} \phi+R_{q} \phi .
$$

Let $\tau \in[0, T]$ be such that

$$
\partial_{t} \tau=-1,
$$

and $\eta$ such that

$$
\phi=(4 \pi \tau)^{-(n+q) / 2} e^{-\eta} .
$$

We then have

$$
\partial_{t} \eta=-\Delta_{\tilde{M}} \eta+|\nabla \eta|^{2}-R_{q}+\frac{n+q}{2 \tau} .
$$

Following [Perelman 2002], the $W$-entropy for the Ricci flow $\tilde{g}(t)$ on the warped product manifold $\tilde{M}$ is defined by

$$
W(\tilde{g}, \eta, \tau)=\int_{\widetilde{M}}\left[\tau\left(|\widetilde{\nabla} \eta|_{\widetilde{M}}^{2}+R_{q}\right)+\eta-(n+q)\right] \phi d \operatorname{vol}_{\tilde{M}},
$$

where $d \operatorname{vol}_{\tilde{M}}$ is the volume form $u d \operatorname{vol}_{M} d \operatorname{vol}_{\mathbb{}} q$ on $(\tilde{M}, \tilde{g})$.

Applying Perelman's [2002] $W$-entropy formula for the Ricci flow to $(\tilde{M}, \tilde{g})$, we have

$$
\frac{d}{d \tau} W(\tilde{g}, \eta, \tau)=-2 \tau \int_{\widetilde{M}}\left|\widetilde{\operatorname{Ric}}+\widetilde{\operatorname{Hess}} \eta-\frac{\tilde{g}}{2 \tau}\right|_{\widetilde{M}}^{2} \phi d \operatorname{vol}_{\widetilde{M}}
$$

By Theorem 2.1, the Laplace-Beltrami on $(\tilde{M}, \tilde{g})$ is given by

$$
\Delta_{\widetilde{M}}=L+u^{-2 / q} \Delta_{\mathbb{T} q},
$$

where

$$
L=\Delta-\nabla \psi \cdot \nabla \text {. }
$$

Here $\Delta$ and $\nabla$ are the Laplace-Beltrami operator and the gradient operator on $(M, g)$, respectively. In the case that $\phi$ is a function depending only on the variable of the horizontal direction, the conjugate heat equation (28) turns out to be the backward heat equation associated with the Witten Laplacian on $(M, g(t))$,

$$
\partial_{t} \phi=-L \phi+R_{q} \phi \text {. }
$$

In this case, $\eta$ is a function depending only on the variable in $M$. Thus,

$$
\begin{aligned}
W(\tilde{g}, \eta, \tau) & =\int_{M \times \mathbb{T} q}\left[\tau\left(|\nabla \eta|^{2}+R_{q}\right)+\eta-(n+q)\right] \phi u d \operatorname{vol}_{M} d \operatorname{vol}_{\mathbb{J} q} \\
& =\int_{M}\left[\tau\left(|\nabla \eta|^{2}+R+2 \Delta \psi-\left(1+\frac{1}{q}\right)|\nabla \psi|^{2}\right)+\eta-(n+q)\right] \phi d \mu .
\end{aligned}
$$


Here $d \mu=u d \operatorname{vol}_{M}$, and we assume $\operatorname{vol}\left(\mathbb{T}^{q}\right)=1$. Note that for any vector field $v$ on $\tilde{M}$, by (8), (9) and (10) we have

$$
\widetilde{\operatorname{Hess}} v=\operatorname{Hess} v-\frac{1}{q} u^{2 / q}\langle\nabla \psi, \nabla v\rangle \sum_{\alpha=1}^{q} d x_{\alpha}^{2} .
$$

Substituting (24) and (31) into (29), we have

$$
\begin{aligned}
\frac{d}{d \tau} W(\tilde{g}, \eta, \tau)= & -2 \tau \int_{M}\left|\widetilde{\operatorname{Ric}}+\widetilde{\operatorname{Hess}} \eta-\frac{\tilde{g}}{2 \tau}\right|_{\tilde{M}}^{2} \phi d \mu \\
= & -2 \tau \int_{M} \mid \operatorname{Ric}_{\psi}^{q}+\operatorname{Hess} \eta-\frac{g}{2 \tau} \\
& \quad+\left.\frac{1}{q}\left(\Delta \psi-|\nabla \psi|^{2}-\langle\nabla \psi, \nabla \eta\rangle-\frac{q}{2 \tau}\right) u^{2 / q} \sum_{\alpha=1}^{q} d x_{\alpha}^{2}\right|_{\tilde{M}} ^{2} \phi d \mu \\
= & -2 \tau \int_{M}\left(\left|\operatorname{Ric}_{\psi}^{q}+\operatorname{Hess} \eta-\frac{g}{2 \tau}\right|^{2}\right. \\
& \left.\quad+\frac{1}{q}\left(\Delta \psi-|\nabla \psi|^{2}-\langle\nabla \psi, \nabla \eta\rangle-\frac{q}{2 \tau}\right)^{2}\right) \phi d \mu .
\end{aligned}
$$

Thus we have proved the following $W$-entropy formula for the backward heat equation associated with the Witten Laplacian on compact manifolds equipped with Lott's modified Ricci flow and time dependent potentials.

Theorem 5.1. Let $(M, g(t), \psi(t))$ be a compact manifold with a family of Riemannian metrics $g(t)$ and potentials $\psi(t)$ which satisfy

$$
\begin{aligned}
& \partial_{t} g=-2\left(\text { Ric }+ \text { Hess } \psi-\frac{1}{q} \nabla \psi \otimes \nabla \psi\right), \\
& \partial_{t} \psi=\Delta \psi-|\nabla \psi|^{2} .
\end{aligned}
$$

Let $d \mu=e^{-\psi} d \operatorname{vol}_{M}$, and $L=\Delta-\nabla \psi \cdot \nabla$. Let $\phi$ be a positive solution to the backward heat equation of the Witten Laplacian on M, i.e.,

$$
\partial_{t} \phi=-L \phi+R_{q} \phi
$$

where $R_{q}=R+2 \Delta \psi-(1+1 / q)|\nabla \psi|^{2}$. Define the W-entropy $W_{q}(g, \psi, \eta, \tau)$ by

$$
W_{q}(g, \psi, \eta, \tau)=\int_{M}\left[\tau\left(|\nabla \eta|^{2}+R_{q}\right)+\eta-(n+q)\right] \phi d \mu,
$$

where $\phi=(4 \pi \tau)^{-(n+q) / 2} e^{-\eta}$, and $(\eta, \tau)$ satisfies

$$
\partial_{t} \eta=-L \eta+|\nabla \eta|^{2}-R_{q}+\frac{n+q}{2 \tau}, \quad \partial_{t} \tau=-1 .
$$


Then

$\frac{d}{d \tau} W_{q}(g, \psi, \eta, \tau)$

$=-2 \tau \int_{M}\left(\left|\operatorname{Ric}_{\psi}^{q}+\operatorname{Hess} \eta-\frac{g}{2 \tau}\right|^{2}+\frac{1}{q}\left(\Delta \psi-|\nabla \psi|^{2}-\langle\nabla \psi, \nabla \eta\rangle-\frac{q}{2 \tau}\right)^{2}\right) \phi d \mu$.

In particular, $W_{q}(g, \psi, \eta, \tau)$ is decreasing in the backward time $\tau$, and the monotonicity is strict unless

$$
\begin{aligned}
\operatorname{Ric}_{\psi}^{q}+\operatorname{Hess} \eta & =\frac{g}{2 \tau}, \\
\Delta \psi-|\nabla \psi|^{2} & =\langle\nabla \psi, \nabla \eta\rangle-\frac{q}{2 \tau} .
\end{aligned}
$$

As an application of the $W$-entropy formula in Theorem 1.3, we have:

Theorem 5.2. Let $(M, g(t), \psi(t))$ be a compact manifold with a family of Riemannian metrics $g(t)$ and potentials $\psi(t)$ which satisfy

$$
\begin{aligned}
& \partial_{t} g=-2\left(\text { Ric }+ \text { Hess } \psi-\frac{1}{q} \nabla \psi \otimes \nabla \psi\right), \\
& \partial_{t} \psi=\Delta \psi-|\nabla \psi|^{2} .
\end{aligned}
$$

Then there exists a positive and smooth function $u=e^{-\eta / 2}$ such that $\eta$ achieves the optimal logarithmic Sobolev constant $\mu(\tau)$ defined by

$$
\mu(\tau):=\inf \left\{W_{q}(g, \psi, \eta, \tau): \int_{M} \frac{e^{-\eta}}{(4 \pi \tau)^{(n+q) / 2}} d \mu=1\right\},
$$

where

$$
W_{q}(g, \psi, \eta, \tau)=\int_{M}\left(\tau\left(|\nabla \eta|^{2}+R_{q}\right)+\eta-(n+q)\right) \phi d \mu,
$$

Indeed, $u=e^{-\eta / 2}$ is a solution to the nonlinear PDE

$$
-4 \tau L u+\tau R_{q} u-2 u \log u-(n+q) u=\mu(\tau) u .
$$

Moreover, $\mu(\tau)$ is decreasing in $\tau$ on $[0, T]$.

Proof. The proof is similar to Perelman's [2002] monotonicity theorem for the $\mu$-invariant for Ricci flow. See also [Cao and Zhu 2006; Chow et al. 2006; Kleiner and Lott 2008; Morgan and Tian 2007] and the proof of Theorem 1.3.

\section{The $W$-entropy formula for the Witten Laplacian with negative Bakry-Émery Ricci curvature}

The $W$-entropy formula (3) only implies the monotonicity of the $W$-entropy for the Witten Laplacian on complete Riemannian manifolds with nonnegative $m$ dimensional Bakry-Émery Ricci curvature, and the $W$-entropy formula (4) only 
implies the monotonicity of the $W$-entropy for the Witten Laplacian on compact Riemannian manifolds with time dependent metrics and potentials satisfying the super $m$-dimensional Bakry-Émery Ricci flow and the conjugate heat equation. On the other hand, J. Li and X. Xu [2011] introduced a $W$-entropy for the heat equation $\partial_{t} u=\Delta u$ on complete Riemannian manifolds with Ricci curvature bounded from below by a negative constant. In this section, we combine the ideas in [ $\mathrm{Li}$ and $\mathrm{Xu}$ 2011; Li 2012; 2014] and Section 4 to extend Theorem 1.1 to the Witten Laplacian on complete Riemannian manifolds with $\operatorname{Ric}_{m, n}(L)$ bounded from below by a negative constant, and extend Theorem 1.2 to the Witten Laplacian on compact Riemannian manifolds with time dependent metrics and potentials satisfying the $K$-super $m$-dimensional Bakry-Émery Ricci flow and the conjugate heat equation.

Recall the following entropy dissipation formulas on complete Riemannian manifolds.

Theorem 6.1 [Li 2012; 2014]. Let $(M, g)$ be a complete Riemannian manifold with the bounded geometry condition, and $\phi \in C^{4}(M)$ with $\nabla \phi \in C_{b}^{3}(M)$. Let $u$ be the fundamental solution to the heat equation $\partial_{t} u=L u$. Let

$$
H(u, t)=-\int_{M} u \log u d \mu .
$$

Then

$$
\frac{d}{d t} H(u, t)=\int_{M} \frac{|\nabla u|^{2}}{u} d \mu
$$

and

$$
\frac{d^{2}}{d t^{2}} H(u, t)=-2 \int_{M}\left(\left|\nabla^{2} \log u\right|^{2}+\operatorname{Ric}(L)(\nabla \log u, \nabla \log u)\right) u d \mu .
$$

Proposition 6.2. Let $m \geq n$ and $K \geq 0$ be constants. Under the same assumptions as in Theorem 6.1, define

$$
H_{m, K}(u, t)=-\int_{M} u \log u d \mu-\frac{m}{2}(1+\log (4 \pi t))-\frac{m}{2} K t\left(1+\frac{K t}{6}\right),
$$

Then

$$
\frac{d}{d t} H_{m, K}(u, t)=\int_{M}\left(\frac{|\nabla u|^{2}}{u^{2}}-\frac{m}{2 t}-\frac{m K}{2}\left(1+\frac{K t}{3}\right)\right) u d \mu .
$$

In particular, if $\operatorname{Ric}_{m, n}(L) \geq-K$, then

$$
\frac{d}{d t} H_{m, K}(u, t) \leq 0,
$$

Proof. By Theorem 6.1, we have

$$
\frac{d}{d t} H_{m, K}(u, t)=\int_{M}\left(\frac{|\nabla u|^{2}}{u^{2}}-\frac{m}{2 t}-\frac{m K}{2}\left(1+\frac{K t}{3}\right)\right) u d \mu .
$$


By the same argument as in [ $\mathrm{Li}$ and $\mathrm{Xu} 2011]$, or using the warped product approach as in [Charalambous and $\mathrm{Lu} \mathrm{2015]} \mathrm{and} \mathrm{the} \mathrm{Li-Yau-type} \mathrm{differential} \mathrm{Harnack} \mathrm{in-}$ equality obtained by $\mathrm{J}$. $\mathrm{Li}$ and $\mathrm{X}$. Xu [2011], we can prove that, if $\mathrm{Ric}_{m, n}(L) \geq-K$, then

$$
\frac{|\nabla u|^{2}}{u^{2}}-\left(1+\frac{2 K t}{3}\right) \frac{\partial_{t} u}{u} \leq \frac{m}{2 t}+\frac{m K}{2}\left(1+\frac{K t}{3}\right) .
$$

From this we may use the fact $\int_{M} \partial_{t} u d \mu=\int_{M} L u d \mu=0$ to conclude

$$
\frac{d}{d t} H_{m, K}(u, t) \leq 0 .
$$

We now prove the main result of this section.

Theorem 6.3. Let $m \geq n$ and $K \geq 0$ be constants. Under the same assumptions as in Theorem 6.1, define the W-entropy by the Boltzmann formula

$$
W_{m, K}(u, t)=\frac{d}{d t}\left(t H_{m, K}(u)\right) \text {. }
$$

Set $u=\frac{e^{-f}}{(4 \pi t)^{m / 2}}$. Then

$$
W_{m, K}(u, t)=\int_{M}\left(t|\nabla f|^{2}+f-m\left(1+\frac{K t}{2}\right)^{2}\right) u d \mu,
$$

and

$$
\begin{aligned}
& \frac{d}{d t} W_{m, K}(u, t)= \\
& -2 t \int_{M}\left(\left|\nabla^{2} f-\left(\frac{1}{2 t}+\frac{K}{2}\right) g\right|^{2}+\left(\operatorname{Ric}_{m, n}(L)+K g\right)(\nabla f, \nabla f)\right) u d \mu \\
& \quad-\frac{2 t}{m-n} \int_{M}\left(\nabla \phi \cdot \nabla f+(m-n)\left(\frac{1}{2 t}+\frac{K}{2}\right)\right)^{2} u d \mu .
\end{aligned}
$$

In particular, if $\operatorname{Ric}_{m, n}(L) \geq-K$, then

$$
\frac{d}{d t} W_{m, K}(u, t) \leq 0 .
$$

Proof. We can prove (32) by direct calculation. By Theorem 6.1, we have

$$
\begin{aligned}
\frac{d}{d t} W_{m, K}(u, t) & =\frac{d}{d t} W(u, t)-\frac{m}{2 t}- \\
=-2 \int_{M} t\left(\left|\nabla^{2} \log u\right|^{2}\right. & +\operatorname{Ric}(L)(\nabla \log u, \nabla \log u)) u d \mu \\
& +2 \int_{M}|\nabla \log u|^{2} u d \mu-\frac{m}{2 t}-m K\left(1+\frac{K t}{2}\right) .
\end{aligned}
$$

Defining

$$
\kappa(t)=K\left(1+\frac{K t}{2}\right), \quad \lambda(u, t)=\left|\nabla^{2} \log u+\frac{g}{2 t}+\frac{K g}{2}\right|^{2},
$$


note that

$2 t\left|\nabla^{2} \log u\right|^{2}+\frac{m}{2 t}+m \kappa(t)=2 t \lambda(u, t)-2(1+K t) \Delta \log u+(m-n)\left(\frac{1}{2 t}+\kappa(t)\right)$.

Integrating by parts yields

$$
\begin{aligned}
\frac{d}{d t} W_{m, K}(u, t)=- & 2 \int_{M} t(\lambda(u, t)+(\operatorname{Ric}(L)+K g)(\nabla \log u, \nabla \log u)) u d \mu \\
& +2(1+K t) \int_{M}(\nabla \log u \cdot \nabla \phi) u d \mu+(m-n)\left(\frac{1}{2 t}+\kappa(t)\right) \\
=- & 2 \int_{M} t\left(\lambda(u, t)+\left(\operatorname{Ric}_{m, n}(L)+K g\right)(\nabla \log u, \nabla \log u)\right) u d \mu \\
& \quad-\frac{2 t}{m-n} \int_{M}\left(\nabla \log u \cdot \nabla \phi-(m-n)\left(\frac{1}{2 t}+\frac{K}{2}\right)\right)^{2} u d \mu .
\end{aligned}
$$

In particular, if $\operatorname{Ric}_{m, n}(L) \geq-K g, W_{m, K}(u, t)$ is monotone decreasing.

Remark 6.4. Suppose that $\operatorname{Ric}_{m, n}(L) \geq-K$. By Theorem 6.3, $\frac{d}{d t} W_{m, K}(u, t)=0$ if and only if

$$
\operatorname{Ric}_{m, n}(L)=-K g, \quad \nabla^{2} f=\left(\frac{1}{2 t}+\frac{K}{2}\right) g, \quad \nabla \phi \cdot \nabla f=-(m-n)\left(\frac{1}{2 t}+\frac{K}{2}\right) .
$$

In particular, if $m=n$ and $\phi=C$ is a constant, then $(M, g)$ is an Einstein manifold with Ric $=-K$, and the potential $f$ satisfies the shrinking gradient Ricci soliton equation (see [ $\mathrm{Li}$ and $\mathrm{Xu} 2011]$ )

$$
\frac{1}{2} \text { Ric }+\nabla^{2} f=\frac{g}{2 t} .
$$

In general, $(M, g)$ is a quasi-Einstein manifold with the metric $g$ such that $\operatorname{Ric}_{m, n}(L)=-K g$, and the potential $f$ satisfies the shrinking gradient quasi-Ricci soliton equation

$$
\frac{1}{2} \operatorname{Ric}_{m, n}(L)+\nabla^{2} f=\frac{g}{2 t} .
$$

Remark 6.5. Similarly to Section 2 , in the case that $m \in \mathbb{N}, m \geq n$ and $M$ is a compact Riemannian manifold, we can give a new proof of Theorem 6.3 via the warped product method. Let $\widetilde{M}=M \times N$, where $\left(N, g_{N}\right)$ is a compact Riemannian manifold with dimension $q=m-n$. Consider the following warped product metric on $\tilde{M}$ :

$$
\tilde{g}=g_{M} \oplus e^{-2 \phi / q} g_{N}
$$


Applying the $W$-entropy formula due to J. Li and X. Xu [2011] for the heat equation $\partial_{t} u=\Delta_{\widetilde{M}} u$ on $(\tilde{M}, \tilde{g})$, we have

$$
\begin{aligned}
& \frac{d}{d t} \widetilde{W}_{m, K}(u, t) \\
& =-2 \int_{\widetilde{M}} t\left(\left|\widetilde{\nabla}^{2} f-\frac{\tilde{g}}{2 t}-\frac{K \tilde{g}}{2}\right|^{2}+(\widetilde{\operatorname{Ric}}+K \tilde{g})(\widetilde{\nabla} \log u, \widetilde{\nabla} \log u)\right) u d \mu d v_{N} .
\end{aligned}
$$

From (11), we get

$$
\begin{aligned}
& \left|\widetilde{\nabla}^{2} f-\frac{\tilde{g}}{2 t}-\frac{K \tilde{g}}{2}\right|^{2} \\
& =\left|\nabla^{2} f-\frac{g}{2 t}-\frac{K g}{2}\right|^{2}+\frac{2}{m-n}\left(\nabla \phi \cdot \nabla f+(m-n)\left(\frac{1}{2 t}+\frac{K}{2}\right)\right)^{2} .
\end{aligned}
$$

On the other hand, by [Besse 1987; Lott 2003; Li 2005], we have

$$
(\widetilde{\operatorname{Ric}}+K \tilde{g})(\widetilde{\nabla} \log u, \widetilde{\nabla} \log u)=\left(\operatorname{Ric}_{m, n}(L)+K g\right)(\nabla \log u, \nabla \log u) .
$$

From (34), (35) and (36), we reprove (33). Note that (35) also gives a natural geometric interpretation of the third term in the $W$-entropy formula (33).

We now extend Theorem 6.3 to the Witten Laplacian on compact manifolds with time dependent metrics and potentials.

Theorem 6.6. Let $m \geq n$ and $K \geq 0$ be constants. Under the same assumptions as in Theorem 1.2, define

$$
H_{m, K}(u, t)=-\int_{M} u \log u d \mu-\frac{m}{2}(1+\log (4 \pi t))-\frac{m}{2} K t\left(1+\frac{K t}{6}\right)
$$

and

$$
W_{m, K}(u, t)=\frac{d}{d t}\left(t H_{m, K}(u)\right) .
$$

Set $u=e^{-f} /(4 \pi t)^{m / 2}$. Then

$$
W_{m, K}(u, t)=\int_{M}\left(t|\nabla f|^{2}+f-m\left(1+\frac{K t}{2}\right)^{2}\right) \frac{e^{-f}}{(4 \pi t)^{m / 2}} d \mu,
$$

and

$$
\begin{aligned}
\frac{d}{d t} W_{m, K}(u, t)=- & 2 t \int_{M}\left|\nabla^{2} f-\frac{g}{2 t}-\frac{K g}{2}\right|^{2} \frac{e^{-f}}{(4 \pi t)^{m / 2}} d \mu \\
& -2 t \int_{M}\left(\frac{1}{2} \frac{\partial g}{\partial t}+\operatorname{Ric}_{m, n}(L)+K g\right)(\nabla f, \nabla f) \frac{e^{-f}}{(4 \pi t)^{m / 2}} d \mu \\
& -\frac{2 t}{m-n} \int_{M}\left(\nabla \phi \cdot \nabla f+(m-n)\left(\frac{1}{2 t}+\frac{K}{2}\right)\right)^{2} \frac{e^{-f}}{(4 \pi t)^{m / 2}} d \mu .
\end{aligned}
$$


In particular, if $\{g(t), \phi(t), t \in(0, T]\}$ is the $K$-super $m$-dimensional Bakry-Émery Ricci flow and satisfies the conjugate equation

$$
\begin{gathered}
\frac{1}{2} \frac{\partial g}{\partial t}+\operatorname{Ric}_{m, n}(L) \geq-K g, \\
\frac{\partial \phi}{\partial t}=\frac{1}{2} \operatorname{Tr}\left(\frac{\partial g}{\partial t}\right),
\end{gathered}
$$

then $W_{m, K}(u, t)$ is decreasing in $t \in(0, T]$, i.e.,

$$
\frac{d}{d t} W_{m, K}(u, t) \leq 0, \quad \text { for all } t \in(0, T] .
$$

Proof. By (19), and replacing $\operatorname{Ric}(L)$ by $\frac{1}{2} \frac{\partial g}{\partial t}+\operatorname{Ric}(L)$, the proof is similar to the proof of Theorem 6.3.

Finally, as an application of Theorem 6.6, we have the following.

Theorem 6.7. Let $(M, g(t), \phi(t), t \in[0, T])$ be as in Theorem 6.6. Then there exists a positive and smooth function $u=e^{-v / 2}$ such that $v$ achieves the optimal logarithmic Sobolev constant $\mu_{K}(t)$ defined by

$$
\mu_{K}(t):=\inf \left\{W_{m, K}(u, t): \int_{M} \frac{e^{-v}}{(4 \pi t)^{m / 2}} d \mu=1\right\} .
$$

Indeed, $u=e^{-v / 2}$ is a solution to the nonlinear PDE

$$
-4 t L u-2 u \log u-m\left(1+\frac{K t}{2}\right)^{2} u=\mu_{K}(t) u .
$$

Moreover, if $\{g(t), \phi(t), t \in[0, T]\}$ satisfies the $K$-super m-dimensional BakryÉmery Ricci flow (37) and the conjugate equation (38), then $\mu_{K}(t)$ is decreasing in $t$ on $[0, T]$.

Proof. The proof is similar to the proof of Theorem 1.3.

\section{Note added in proof}

In a recent preprint, the authors introduced the $W$-entropy and proved the $W$-entropy formula for the heat equation of the Witten Laplacian on complete Riemannian manifolds with the $\operatorname{CD}(K, \infty)$ condition (i.e., $\operatorname{Ric}(L) \geq K)$ and extended the corresponding result to the heat equation of the time dependent Witten Laplacian on compact Riemannian manifolds equipped with the $K$-super Perelman Ricci flow with respect to the Bakry-Émery Ricci curvature (i.e., $\frac{1}{2} \frac{\partial g}{\partial t}+\operatorname{Ric}(L) \geq K g$ ). See [Li and Li 2014a].

\section{Acknowledgements}

This paper is an extended version of the preprint [ $\mathrm{Li}$ and $\mathrm{Li} 2014 \mathrm{~b}]$. We would like to thank Prof. D. Bakry, Prof. B.-L. Chen and Dr. Yu-Zhao Wang for their interest 
and useful discussions, and Prof. Zhiqin Lu for the citation of the first version of this paper in [Charalambous and Lu 2015]. We are very grateful to the anonymous referees for their careful reading and helpful comments for the revision of the paper.

\section{References}

[Andrews and Ni 2012] B. Andrews and L. Ni, "Eigenvalue comparison on Bakry-Emery manifolds", Comm. Partial Differential Equations 37:11 (2012), 2081-2092. MR 3005536 Zbl 1258.35153

[Bakry and Émery 1985] D. Bakry and M. Émery, "Diffusions hypercontractives", pp. 177-206 in Séminaire de probabilités, XIX, 1983/84, edited by J. Azéma and M. Yor, Lecture Notes in Mathematics 1123, Springer, Berlin, 1985. MR 88j:60131 Zbl 0561.60080

[Bakry and Ledoux 2006] D. Bakry and M. Ledoux, "A logarithmic Sobolev form of the Li-Yau parabolic inequality”, Rev. Mat. Iberoam. 22:2 (2006), 683-702. MR 2008m:58051 Zbl 1116.58024

[Bakry and Qian 1999] D. Bakry and Z. M. Qian, "Harnack inequalities on a manifold with positive or negative Ricci curvature", Rev. Mat. Iberoamericana 15:1 (1999), 143-179. MR 2000f:58052 Zbl 0924.58096

[Bakry and Qian 2000] D. Bakry and Z. Qian, "Some new results on eigenvectors via dimension, diameter, and Ricci curvature", Adv. Math. 155:1 (2000), 98-153. MR 2002g:58048 Zbl 0980.58020

[Besse 1987] A. L. Besse, Einstein manifolds, Ergebnisse der Mathematik (3) 10, Springer, Berlin, 1987. MR 88f:53087 Zbl 0613.53001

[Cao and Zhu 2006] H.-D. Cao and X.-P. Zhu, "A complete proof of the Poincaré and geometrization conjectures-application of the Hamilton-Perelman theory of the Ricci flow", Asian J. Math. 10:2 (2006), 165-492. MR 2008d:53090 Zbl 1200.53057

[Chang et al. 2011] S.-C. Chang, T.-J. Kuo, and S.-H. Lai, "Li-Yau gradient estimate and entropy formulae for the CR heat equation in a closed pseudohermitian 3-manifold", J. Differential Geom. 89:2 (2011), 185-216. MR 2863916 Zbl 1264.32030

[Charalambous and Lu 2015] N. Charalambous and Z. Lu, "Heat kernel estimates and the essential spectrum on weighted manifolds", J. Geom. Anal. 25:1 (2015), 536-563. MR 3299292 Zbl 06406917

[Chow et al. 2006] B. Chow, P. Lu, and L. Ni, Hamilton's Ricci flow, vol. 77, Graduate Studies in Mathematics, American Mathematical Society, Providence, RI, 2006. MR 2008a:53068 Zbl 1118:53001

[Ecker 2007] K. Ecker, “A formula relating entropy monotonicity to Harnack inequalities", Comm. Anal. Geom. 15:5 (2007), 1025-1061. MR 2009c:53088 Zbl 1167.53055

[Fang et al. 2009] F. Fang, X.-D. Li, and Z. Zhang, "Two generalizations of Cheeger-Gromoll splitting theorem via Bakry-Émery Ricci curvature", Ann. Inst. Fourier (Grenoble) 59:2 (2009), 563-573. MR 2010b:53057 Zbl 1166.53023

[Futaki et al. 2013] A. Futaki, H. Li, and X.-D. Li, "On the first eigenvalue of the Witten-Laplacian and the diameter of compact shrinking solitons", Ann. Global Anal. Geom. 44:2 (2013), 105-114. MR 3073582 Zbl 1273.58018

[Guo et al. 2015] H. Guo, R. Philipowski, and A. Thalmaier, "An entropy formula for the heat equation on manifolds with time-dependent metric, application to ancient solutions", Potential Anal. 42:2 (2015), 483-497. MR 3306693 Zbl 1309.53051

[Huang and Li 2014] G. Huang and H. Li, "Gradient estimates and entropy formulae of porous medium and fast diffusion equations for the Witten Laplacian”, Pacific J. Math. 268:1 (2014), 47-78. MR 3207600 Zbl 1297.35059 
[Kleiner and Lott 2008] B. Kleiner and J. Lott, "Notes on Perelman's papers", Geom. Topol. 12:5 (2008), 2587-2855. MR 2010h:53098 Zbl 1204.53033

[Kotschwar and Ni 2009] B. Kotschwar and L. Ni, "Local gradient estimates of $p$-harmonic functions, 1/H-flow, and an entropy formula", Ann. Sci. Éc. Norm. Supér. (4) 42:1 (2009), 1-36. MR 2010g:53121 Zbl 1182.53060

[Li 2005] X.-D. Li, "Liouville theorems for symmetric diffusion operators on complete Riemannian manifolds", J. Math. Pures Appl. (9) 84:10 (2005), 1295-1361. MR 2006f:58046 Zbl 1082.58036

[Li 2007] X.-D. Li, On Perelman's W-entropy functional on Riemannian manifolds with weighted volume measure, in Thèse d'Habilitation à Diriger des Rechercher, Université Paul Sabatier, 2007.

[Li 2011] X.-D. Li, "Perelman's $W$-entropy for the Fokker-Planck equation over complete Riemannian manifolds", Bull. Sci. Math. 135:6-7 (2011), 871-882. MR 2012m:53077 Zbl 1230.82039

[Li 2012] X.-D. Li, "Perelman's entropy formula for the Witten Laplacian on Riemannian manifolds via Bakry-Emery Ricci curvature”, Math. Ann. 353:2 (2012), 403-437. MR 2915542 Zbl 1280.58016

[Li 2014] X.-D. Li, "Hamilton's Harnack inequality and the $W$-entropy formula on complete Riemannian manifolds", 2014. To appear in Stochastic Process. Appl. arXiv 1303.1242

[Li and Li 2014a] S. Li and X.-D. Li, "Harnack inequalities and W-entropy formula for Witten Laplacian on Riemannian manifolds with $K$-super Perelman Ricci flow", preprint, 2014. arXiv 1412.7034

[Li and Li 2014b] S. Li and X.-D. Li, "W-entropy formula for the Witten Laplacian on manifolds with time dependent metrics and potentials", preprint, 2014. arXiv 1303.6019

[Li and Xu 2011] J. Li and X. Xu, "Differential Harnack inequalities on Riemannian manifolds I: linear heat equation”, Adv. Math. 226:5 (2011), 4456-4491. MR 2012h:53079 Zbl 1226.58009

[Lott 2003] J. Lott, "Some geometric properties of the Bakry-Émery-Ricci tensor", Comment. Math. Helv. 78:4 (2003), 865-883. MR 2004i:53044 Zbl 1038.53041

[Lott 2009] J. Lott, "Optimal transport and Perelman's reduced volume”, Calc. Var. Partial Differential Equations 36:1 (2009), 49-84. MR 2011b:53160 Zbl 1171.53318

[Lu et al. 2009] P. Lu, L. Ni, J.-L. Vázquez, and C. Villani, "Local Aronson-Bénilan estimates and entropy formulae for porous medium and fast diffusion equations on manifolds", J. Math. Pures Appl. (9) 91:1 (2009), 1-19. MR 2010j:35563 Zbl 1156.58015

[Morgan and Tian 2007] J. Morgan and G. Tian, Ricci flow and the Poincaré conjecture, Clay Mathematics Monographs 3, American Mathematical Society, Providence, RI, 2007. MR 2008d:57020 Zbl 1179.57045

[Ni 2004a] L. Ni, "Addenda to: "The entropy formula for linear heat equation"”, J. Geom. Anal. 14:2 (2004), 369-374. MR 2004m:53118b Zbl 1062.58028

[Ni 2004b] L. Ni, “The entropy formula for linear heat equation”, J. Geom. Anal. 14:1 (2004), 87-100. MR 2004m:53118a Zbl 1044.58030

[Otto 2001] F. Otto, "The geometry of dissipative evolution equations: the porous medium equation", Comm. Partial Differential Equations 26:1-2 (2001), 101-174. MR 2002j:35180 Zbl 0984.35089

[Otto and Villani 2000] F. Otto and C. Villani, "Generalization of an inequality by Talagrand and links with the logarithmic Sobolev inequality”, J. Funct. Anal. 173:2 (2000), 361-400. MR 2001k:58076 Zbl 0985.58019

[Perelman 2002] G. Perelman, "The entropy formula for the Ricci flow and its geometric applications", preprint, 2002. arXiv math/0211159

[von Renesse and Sturm 2005] M.-K. von Renesse and K.-T. Sturm, "Transport inequalities, gradient estimates, entropy, and Ricci curvature”, Comm. Pure Appl. Math. 58:7 (2005), 923-940. MR 2006j:53048 Zbl 1078.53028 
[Rothaus 1981] O. S. Rothaus, "Logarithmic Sobolev inequalities and the spectrum of Schrödinger operators", J. Funct. Anal. 42:1 (1981), 110-120. MR 83f:58080b Zbl 0471.58025

[Sturm 2005] K.-T. Sturm, "Convex functionals of probability measures and nonlinear diffusions on manifolds", J. Math. Pures Appl. (9) 84:2 (2005), 149-168. MR 2005k:49126 Zbl 1259.49074

[Villani 2003] C. Villani, Topics in optimal transportation, Graduate Studies in Mathematics 58, American Mathematical Society, Providence, RI, 2003. MR 2004e:90003 Zbl 1106.90001

[Villani 2009] C. Villani, Optimal transport: Old and new, Grundlehren der Mathematischen Wissenschaften 338, Springer, Berlin, 2009. MR 2010f:49001 Zbl 1156.53003

[Wang and Chen 2014] Y. Wang and W. Chen, "Gradient estimates and entropy monotonicity formula for doubly nonlinear diffusion equations on Riemannian manifolds", Math. Methods Appl. Sci. 37:17 (2014), 2772-2781. MR 3271122 Zbl 06446828

[Wang et al. 2013] Y. Wang, J. Yang, and W. Chen, "Gradient estimates and entropy formulae for weighted $p$-heat equations on smooth metric measure spaces", Acta Math. Sci. Ser. B Engl. Ed. 33:4 (2013), 963-974. MR 3072133 Zbl 1299.58018

[Wei and Wylie 2009] G. Wei and W. Wylie, "Comparison geometry for the Bakry-Emery Ricci tensor”, J. Differential Geom. 83:2 (2009), 377-405. MR 2011a:53064 Zbl 1189.53036

Received November 2, 2014. Revised March 24, 2015.

\section{SONGZI LI}

SCHOOL OF MATHEMATICAL SCIENCES

FUDAN UNIVERSITY

220 HANDAN ROAD

SHANGHAI 200433

CHINA

and

Institut de Mathématiques De Toulouse

UNIVERSITÉ PAUL SABATIER

118 ROUTE DE NARBONNE

31062 Toulouse CedeX 9

FRANCE

Songzi.Li@math.univ-toulouse.fr

XIANG-DONG LI

ACAdemy of Mathematics and Systems Science

ChINESE ACADEMY OF SCIENCES

55 ZHONGGUANCUN EAST ROAD

BEIJING 100190

CHINA

xdli@amt.ac.cn 


\title{
PACIFIC JOURNAL OF MATHEMATICS
}

\author{
msp.org/pjm
}

Founded in 1951 by E. F. Beckenbach (1906-1982) and F. Wolf (1904-1989)

\section{EDITORS}

Don Blasius (Managing Editor)

Department of Mathematics

University of California

Los Angeles, CA 90095-1555

blasius@math.ucla.edu

\author{
Paul Balmer \\ Department of Mathematics \\ University of California \\ Los Angeles, CA 90095-1555 \\ balmer@math.ucla.edu \\ Robert Finn \\ Department of Mathematics \\ Stanford University \\ Stanford, CA 94305-2125 \\ finn@math.stanford.edu \\ Sorin Popa \\ Department of Mathematics \\ University of California \\ Los Angeles, CA 90095-1555 \\ popa@math.ucla.edu
}

\author{
Vyjayanthi Chari \\ Department of Mathematics \\ University of California \\ Riverside, CA 92521-0135 \\ chari@math.ucr.edu \\ Kefeng Liu \\ Department of Mathematics \\ University of California \\ Los Angeles, CA 90095-1555 \\ liu@math.ucla.edu \\ Jie Qing \\ Department of Mathematics \\ University of California \\ Santa Cruz, CA 95064 \\ qing@ cats.ucsc.edu
}

\section{PRODUCTION}

Silvio Levy, Scientific Editor, production@msp.org

\section{SUPPORTING INSTITUTIONS}

ACADEMIA SINICA, TAIPEI

CALIFORNIA INST. OF TECHNOLOGY

INST. DE MATEMÁTICA PURA E APLICADA

KEIO UNIVERSITY

MATH. SCIENCES RESEARCH INSTITUTE

NEW MEXICO STATE UNIV.

OREGON STATE UNIV.

\author{
STANFORD UNIVERSITY \\ UNIV. OF BRITISH COLUMBIA \\ UNIV. OF CALIFORNIA, BERKELEY \\ UNIV. OF CALIFORNIA, DAVIS \\ UNIV. OF CALIFORNIA, LOS ANGELES \\ UNIV. OF CALIFORNIA, RIVERSIDE \\ UNIV. OF CALIFORNIA, SAN DIEGO \\ UNIV. OF CALIF., SANTA BARBARA
}

\author{
Daryl Cooper \\ Department of Mathematics \\ University of California \\ Santa Barbara, CA 93106-3080 \\ cooper@math.ucsb.edu \\ Jiang-Hua Lu \\ Department of Mathematics \\ The University of Hong Kong \\ Pokfulam Rd., Hong Kong \\ jhlu@maths.hku.hk \\ Paul Yang \\ Department of Mathematics \\ Princeton University \\ Princeton NJ 08544-1000 \\ yang@math.princeton.edu
}

These supporting institutions contribute to the cost of publication of this Journal, but they are not owners or publishers and have no responsibility for its contents or policies.

See inside back cover or msp.org/pjm for submission instructions.

The subscription price for 2015 is US \$420/year for the electronic version, and \$570/year for print and electronic.

Subscriptions, requests for back issues and changes of subscribers address should be sent to Pacific Journal of Mathematics, P.O. Box 4163, Berkeley, CA 94704-0163, U.S.A. The Pacific Journal of Mathematics is indexed by Mathematical Reviews, Zentralblatt MATH, PASCAL CNRS Index, Referativnyi Zhurnal, Current Mathematical Publications and Web of Knowledge (Science Citation Index).

The Pacific Journal of Mathematics (ISSN 0030-8730) at the University of California, c/o Department of Mathematics, 798 Evans Hall \#3840, Berkeley, CA 94720-3840, is published twelve times a year. Periodical rate postage paid at Berkeley, CA 94704, and additional mailing offices. POSTMASTER: send address changes to Pacific Journal of Mathematics, P.O. Box 4163, Berkeley, CA 94704-0163.

PJM peer review and production are managed by EditFLOW ${ }^{\circledR}$ from Mathematical Sciences Publishers.

\section{PUBLISHED BY}

\section{mathematical sciences publishers \\ nonprofit scientific publishing}

http://msp.org/

(C) 2015 Mathematical Sciences Publishers 


\section{PACIFIC JOURNAL OF MATHEMATICS}

Volume $278 \quad$ No. $1 \quad$ November 2015

Growth tight actions

Goulnara N. Arzhantseva, Christopher H. Cashen and JING TAO

A flag structure on a cusped hyperbolic 3-manifold

ELISHA FALBEL and RAFAEL SANTOS THEBALDI

A new upper bound for the Dirac operators on hypersurfaces

Nicolas GinouX, GeORGES Habib and Simon RaUlot

Games and elementary equivalence of $\mathrm{II}_{1}$-factors

ISAAC GOLDBRING and THOMAS SINCLAIR

Grossberg-Karshon twisted cubes and hesitant walk avoidance

MEGUMI HARADA and EUNJEONG LEE

Gamma factors of distinguished representations of $\mathrm{GL}_{n}(\mathbb{C})$

ALEXANDER KEMARSKY

The $W$-entropy formula for the Witten Laplacian on manifolds with 173 time dependent metrics and potentials

SONGZI LI and XIANG-DONG LI

A diagrammatic categorification of the affine $q$-Schur algebra $\hat{\boldsymbol{S}}(n, n) 201$ for $n \geq 3$

MARCO MACKAAY and ANNE-LAURE THIEL

Showing distinctness of surface links by taking 2-dimensional braids

INASA NAKAMURA

Correction to Modular $L$-values of cubic level 